\title{
Fundamentos de la Ciencia de la Información: De los ordenadores y la física cuántica a las células, sistemas nerviosos y sociedades
}

\author{
Pedro C. Marijuán \\ Departamento Ingeniería Eléctrica, Electrónica y Comunicaciones \\ CPS, Universidad de Zaragoza \\ E 50015 Zaragoza
}

\subsection{Resumen}

El presente artículo reproduce la sesión de apertura de la Primera Conferencia sobre Fundamentos de la Ciencia de la Información celebrada en Madrid, Julio 1994, organizada por la Universidad Carlos III y la Universidad de Zaragoza (véanse las Actas en BioSystems, vol. 38, 1996). Dicha conferencia ha discutido la posibilidad de rescatar la información como herramienta científica central y que incluso llegue a servir de base para un nuevo desarrollo disciplinar. Como el subtítulo de la conferencia indica, la información es entendida aquí en relación con una vasta red de procesos, potencialmente abarcando la integración de ocurrencias físicas (cuánticas), computacionales, celulares, neuronales y sociales. En vez de la discusión sobre un único concepto particular, la "ciencia de la información" aparece como una búsqueda intelectual encaminada a desarrollar una ciencia vertical conectando las diferentes escalas de los "procesos informacionales", implicando a la vez un enfoque unificador y multiperspectivista. Puede trazarse un paralelo con el propio concepto de física, que de un término pre-Galileano particularizado derivó hacia una ciencia vertical, conectando las hasta entonces separadas esferas de los fenómenos celestiales, sublunares y terrestres. Desde este punto de vista más amplio, es interesante que cabe relacionar el "sentido" de la información con el fenómeno de la ausencia (vacíos funcionales o roturas de simetría). (Autor)

Palabras clave: Información. Ciencia de la Información. Significado. Ausencia. Vacío funcional. Perspectivismo.

\subsection{Abstract}

This article reproduces the opening session of the Madrid Conference on

Scire. 2 : 1 (en.-jun. 1996). 
"Foundations of Information Science," organized by the university Carlos III of Madrid and the university of Zaragoza, in July 1994 (see Proceedings in BioSystems, vol. 38, 1966). This conference has been an attempt to rescue information as a central scientific tool and to put it into a new context so as to serve as a basis for a fundamental disciplinary development. The novelty of the conference was that, instead of trying a precise "atomic" definition of information, and actually getting quagmired in it, information was understood as related to a widespread network of processes potentially involving the integration of cosmic (subatomic), molecular, cellular (neuronal), computational, human and social occurrences, demanding both a unifying and a multiperspectivistic approach. So to speak, instead of the discussion of a single particularized concept, information becomes the intellectual adventure of developing a "vertical" science connecting the different scales of "informational processes"-reminding physics itself, which from a pre-Galilean particularized term evolved towards a vertical science connecting the previously separated "celestial", "sublunar", and "terrestrial" occurrences. In this enlarged context, it is interesting that the meaning of information can be discussed in relation with the phenomenon of absence (functional voids or symmetry breaking). (Author)

Keywords: Information. Information Science. Meaning. Absence. Functional Void. Perspectivism.

\section{Introducción y agradecimientos: los origenes de la conferencia}

Esta primera conferencia sobre Fundamentos de la Ciencia de la Información, como muchos de los asistentes a la misma saben bien, ha tenido tras de sí su pequeña historia particular. Surgió como proyecto formal a mediados de 1991, en Chicago, durante una conversación entre Michael Conrad y este autor en un restaurante libanés. Al año siguiente, ya se estaba desarrollando el embrión de una red de cooperación intelectual entre científicos de distintas disciplinas atraídos por la aventura. Durante 1992 y 1993 se presentaron por parte de este grupo de científicos diversas solicitudes de financiación a fundaciones internacionales y españolas potencialmente interesadas en el tema. Sin embargo, a pesar de las positivos comentarios y del indudable interés, no se consiguió que ningún tipo de apoyo efectivo llegara a materializarse.

La situación cambió en el otoño de 1993, cuando un animoso profesor de la universidad Carlos III de Madrid, Fivos Panetsos, se comprometió personalmente a sacar adelante la financiación y organización de la conferencia. Sus gestiones pronto fructificaron y se pudo realizar la convocatoria pública. Poco más tarde, la profesora Araceli Sanchis se encargó de coordinar la logística del evento. No cabe duda que el buen trabajo de ambos es lo que ha hecho posible que hoy nos reunamos aquí. Ambos merecen toda nuestra gratitud.

Scire. 2 : 1 (en.-jun. 1996). 
El grupo de científicos que ha contribuido a la preparación de esta conferencia está integrado, aparte de por Michael Conrad (universidad Wayne State) y Pedro C. Marijuán (universidad de Zaragoza), por Koichiro Matsuno (biofísico, universidad de Nagaoka), Tom Stonier (físico informacional, universidad de Bradford), Johan De Vree (derecho internacional, universidad de Utretch), Antonio Prevosti (biólogo, universidad de Barcelona), Efim Liberman (neurofisiólogo, universidad de Moscú), Ramón Margalef (ecólogo, universidad de Barcelona), y algunos otros de posterior incorporación: Peter Erdi, Peter Arhem, George Kampis, Gukio Gunji, Grupo Forma (Lisboa), etc. El Laboratorio de Inteligencia Artificial de la universidad Carlos III de Madrid, la OTRI de la universidad Carlos III, el Ministerio de Educación (DGICYT) y la Comunidad de Madrid (Consejería de cultura) generosamente han proporcionado los fondos necesarios para la realización de la conferencia. Por parte de la universidad de Zaragoza han apoyado el Vicerrectorado de Investigación, la Fundación Circe, y el Area de Tecnología Electrónica del CPS. Nuestro más sincero agradecimiento a todos ellos.

\section{La reciente historia del termino información: un legado contra- dictorio}

El objetivo de esta conferencia, el motivo por el que ha sido convocada, es el de valorar las posibilidades actuales de transcender las barreras clásicas que han bloqueado el concepto de información. Durante los cuatro próximos días debatiremos aquí el contenido y las paradojas de uno de los conceptos más oscuros y problemáticos y que más se ramifica a través de las más diversas disciplinas. Es curioso que nadie parece poner en duda la importancia y centralidad de este concepto en el presente sistema de las ciencias, pero que a la vez nadie haya llegado a establecer un marco de pensamiento consistente alrededor del mismo.

Como no podía ser menos, la historia contemporánea del concepto es a la vez brillante y descorazonadora. El término información hizo su entrada triunfal en el sistema de las ciencias en la postguerra, a través de la cibernética, la teoría de sistemas, la propia teoría de la información, el desarrollo de los ordenadores, la inmediata aparición de la inteligencia artificial, la nueva linguística, etc. Verdaderamente una atmósfera intelectual de tremendo optimismo se generó alrededor del concepto. En 1941, en una carta a von Neumann, R. Ortway captura el estilo de pensamiento que se estaba gestando:

"Hoy día todo el mundo está hablando de organización, totalidad. Las máquinas de computación, la interconexión automática de teléfonos, los equipos de alta tensión, como los transformadores en cascada, lo mismo que los equipos radiofónicos de emisión y recepción, y también una planta industrial o una oficina son todos ellos ejemplos técnicos de tales organizaciones. Yo creo que en todos existe un elemento común

Scire. 2 : 1 (en.-jun. 1996). 
que es susceptible de formalización.” (Nagy et al., 1989, p. 188)

Información, claramente iba a ser la respuesta. En 1948, Norbert Wiener, fundador de la cibernética, escribía con toda rotundidad: "El papel de la información y la técnica de medir y transmitir la información constituyen una disciplina conjunta para el ingeniero, el fisiólogo, y también para el sociólogo.” (p. vii). Y también, "La información es información, no materia y energía." (p. 132).

Pero posiblemente no hubo suficiente tiempo o posibilidades efectivas de maduración, y las disciplinas y teorías avanzaron dejando demasiados vacíos entre sí. De algún modo, el concepto quedó envuelto en excesivas interconexiones escasamente clarificadas. Mirando hacia atrás es fácil apreciar un proceso de degradación conceptual y pérdida de confianza, incluso ya a principios de los 60, cuando se proclamó formalmente una "ciencia de la información", que sin embargo fue pronto abandonada y absorbida por la entonces emergente inteligencia artificial (véase Marijuán, 1996; véanse también: McLuhan, 1964; Simon, 1969; Brookes, 1974; Scarrot, 1986; Horgan, 1995). Progresivamente, a pesar de su tremendo potencial, el concepto de información fue percibido por las nuevas generaciones de científicos que entraban a estas avenidas interdiciplinares más como una fuente de equívoco y desorientación que como una fuente de esclarecimiento.

Hoy día, tras casi cuatro décadas transcurridas desde su triunfal entrada en las ciencias, una cierta sensación de cansancio y desilusión es común entre los científicos y pensadores que durante largo tiempo han estado interesados en el concepto. Un reciente comentario del físico E. Wigner (1989) puede ilustrar el cambio de actitud que se ha producido:

"La presente teoría de la información es por completo atípica. Y creo que ya es hora de cambiarla. Pero no me ha ido nada bien con ella. No he podido conseguirlo. Quizás no debería hablar de ésto, porque no está bien reconocer que uno intenta hacer algo y no lo consigue." (p. 256)

Verdaderamente, durante los cuarenta años transcurridos desde su aparición formal, la información es como si se hubiese guiado por aquel viejo adagio de "hacer de aprendiz de todo" ("y de oficial de nada"). Durante este tiempo se ha visto envuelta en aspectos y disciplinas tan dispares como:

- la formulación del segundo principio de la termodinámica y el concepto de entropía (p.ej., las discusiones acerca del “demonio de Maxwell”),

- el problema de la medida en mecánica cuántica,

- por supuesto, la teoría de la información de Shanon,

- los sistemas fuera del equilibrio y la dinámica de sistemas no-lineales

Scire. 2 : 1 (en.-jun. 1996). 
- el ADN y el procesamiento de las enzimas

- la evolución biológica y el estatus de la teoría darwinista

- la medida de la diversidad ecológica,

- los orígenes y evolución del sistema nervioso

- el funcionamiento del cerebro,

- la naturaleza de la inteligencia,

- la lógica (profundidad lógica, complejidad algorítmica),

- la linguística (significado, semántica),

- los fundamentos de la ontología y epistemología,

- el paradigma representacional de la inteligencia artificial,

- la electrónica y la ingeniería de sistemas,

- los medios de comunicación de masas y las nuevas tecnologías informacionales,

- la gestión de la documentación y la biblioteconomía,

- los fundamentos de la economía y las ciencias sociales,

- la filosofía política...

El uso del término información en todos esos campos no es en sí incorrecto, por supuesto, pero su coherencia global prácticamente ha desaparecido. Diríase que cualquier aspecto de la vida humana, o del comportamiento de los sistemas biológicos, o incluso de la evolución cósmica, contiene y procesa información.

\section{3. ¿Que hacer? Objetivos de la conferencia}

Frente a este confuso panorama, la búsqueda de una mayor claridad, a la luz de cambios significativos en algunos de los anteriores campos, ha sido la razón principal para convocar esta conferencia. La muy buena aceptación que hemos encontrado, hasta entusiasta en algunos casos, por parte de los científicos y estudiosos con quienes hemos entrado en contacto, ha robustecido nuestra confianza en las posibilidades de esta conferencia. Como se sugería en el manifiesto-presentación inicialmente preparado, muchos de nosotros compartimos la impresión de que actualmente han surgido importantes nuevas posibilidades para avanzar en la consolidación del concepto (la "ciencia"). Principalmente debido a:

- las nuevas áreas areas de investigación en biología molecular y biofísica,

- las nuevas orientaciones en computación y "ciencias de la complejidad,"

- los nuevos esquemas de pensamiento en las neurociencias, 
- y los intensos debates en filosofía, economía, ciencias sociales y políticas acerca de orden, caos, emergencia, auto-organización, complejidad, etc.

El objetivo central que inicialmente se ha planteado esta conferencia es bosquejar una casa común, un "paraguas interdisciplinar" donde pueda tener lugar la fertilización e intercambio mutuo entre estos nuevos enfoques. De hecho no existen foros actuales en que se produzca ese tipo de interacción: la presencia sistemática de lo molecular junto a lo neuro-computacional y lo social. Y quizás esa fecundación mutua sea una necesidad de primer orden. Si el punto de vista esbozado en la presentación-manifiesto de esta convocatoria es correcto, la nueva perspectiva a construir no seguiría un modelo "reduccionista", siendo obtenida a partir de un nivel único o preferente de análisis, sino que habría de avanzar en paralelo cubriendo las tres "sociedades informacionales" fundamentales: la "sociedad de las enzimas", la "sociedad de las células (neuronas)" y la "sociedad de los individuos." Teniendo en cuenta sobre todo que ninguna de esas "sociedades" tomada aisladamente proporciona una visión completa del fenómeno de la información. Cada una proporciona una serie de claves específicas importantes, pero ninguna de esas "sociedades" en solitario, ninguna disciplina aislada, parece que puede llegar a resolver los enigmas del concepto de la información.

¿Cómo podrían avanzar y cohesionarse mútuamente tan diferentes perspectivas? Ese es el experimento que vamos a seguir durante estos días. Sin duda requerirá unas buenas dosis de paciencia, sentido común e imaginación. Posiblemente el mejor resultado del experimento sería que esta conferencia pudiera continuar sus esfuerzos, es decir que en próximos años volviera a reunirse.

\section{Antecedentes históricos}

A modo de referente, cabría apuntar a un par de antecedentes históricos acerca de periodos complicados en la historia de la ciencia que, de algún modo, están relacionados con el objetivo de esta empresa.

El primero de ellos se produjo en el siglo XVI. Tuvo que ver con el propio concepto de "física". Durante aquel siglo que ahora idealizamos bajo la etiqueta de Renacimiento también existía un ambiente de profunda crisis social e intelectual. Intensos debates se estaban produciendo entre la "vieja guardia" aristotélico-escolástica y los seguidores de la "nueva ciencia": Erasmo, Luis Vives, Pierre Ramus, Thomas More, Francis Bacon. En relación con la física, buena parte de los debates estaban centrados en la física entendida como algo, como una sustancia subyaciendo todos los fenómenos naturales. Como sabemos bien, particularmente tras los desarrollos de Galileo y Newton, la física iba a abandonar su carácter de concepto referido a una sustancia particular y pasar a ser una esplén-

Scire. 2 : 1 (en.-jun. 1996). 
dida ciencia abarcando un enorme abanico de procesos diferentes.

Pues bien, la sospecha es que con el término información puede que estemos en puertas de que ocurra algo parecido. Lo que hoy en día estamos denominando bajo la etiqueta de información podría constituir no un mero concepto sino implicar una enorme red de procesos interrelacionados. ¿Qué tipo de procesos? Siguiendo las orientaciones de Conrad $(1986,1989)$ sobre el flujo vertical de "procesos informacionales" interrelacionados-de moléculas a células, organismos y ecosistemas - podemos imaginar un flujo paralelo de información comprendiendo las sociedades humanas también. De los paises y sistemas socioeconómicos en la parte más alta, a las instituciones e individuos, y entonces a los sistemas neuronales, a las células y a las moléculas en la base inferior. ¿Cómo podrían delinearse los hitos fundamentales de semejante flujo de información entre tan diferentes "sociedades"? Enfaticemos que el flujo de energía de los fotones solares a través de los distintos niveles de los ecosistemas fué delineado no por la discusión de un único concepto, sino porque una poderosa disciplina científica, la física, se fue desarrollando y trabajando eficazmente.

Ese quizás es el mensaje más importante de ésta anécdota histórica: enfatizar la necesidad de desarrollar una genuina disciplina científica, y no quedar paralizados en la discusión de una única etiqueta conceptual llena de paradojas.

La otra anécdota histórica nos lleva a Toledo, entre los siglos XII y XIII. De hecho nos conduce a las discusiones acerca de los números arábigos, y particularmente a la cifra conocida como cero. Los números fueron un problema sin resolver para griegos y romanos, y también para los matemáticos de la Europa medieval. No pudieron desarrollar verdaderos sistemas de numeración posicional porque no incluían el cero. Entonces se hacía imposible la realización de sumas y multiplicaciones sin un ábaco y grandes habilidades. Sólo un puñado de calculistas eran capaces de hacer una división "a mano".

Atendamos a que tras el nacimiento de la ciencia y filosofía griegas, se necesitaron casi 15 siglos para que los estudiosos europeos cayeran en la cuenta de la necesidad de representar la ausencia como caso en sí, y fue en Córdoba, Toledo y Sicilia donde este conocimiento pasó de fuentes árabes a los estudiosos occidentales. En su raiz, el cero es la representación de la ausencia como caso propio. Fue descubierto por mercaderes y filósofos indios en los siglos V y VI (a.d.). Existe discusión escrita acerca del uso del cero en documentos del siglo VII. Estaba relacionado con un punto de vista filosófico muy sofisticado acerca de la negación y la ausencia. Aparentemente era un insignificante nuevo símbolo, pero su repercusión futura sería enorme. A este respecto escribe Whitehead (1948) acerca de la importancia del símbolo cero:

Al liberar al cerebro de todo trabajo innecesario, una buena anotación per-

Scire. 2 : 1 (en.-jun. 1996). 
mite que se concentre en problemas más avanzados, y de hecho aumenta el poder mental de la raza... La civilización avanza al extenderse el número de operaciones importantes que podemos llevar a cabo sin tener que pensar a cerca de ellas. Las operaciones de pensamiento son como las cargas de caballería en una batalla: Están estrictamente limitadas en número, requieren caballos frescos, y deben hacerse en los momentos decisivos.” (pp.39, 42)

La idea misma de ausencia puede ser crucial también para las discusiones sobre la información; puede proporcionar una clave estratégica para adentrarse en las interioridades de las mencionadas "sociedades" informacionales. Como la reciente biología molecular ha puesto de manifiesto (Marijuán, 1996b), la degradación de proteínas y la muerte celular son factores necesarios para el desarrollo tanto de la célula como del organismo. Cabe argumentar que partiendo de dicha degradación (y la acoplada síntesis) de proteínas, conectando con la dinámica del ciclo y la muerte celular, continuando con el ciclo global del organismo, y finalmente acudiendo a la dinámica de los sistemas socio-económicos, podemos apreciar en todos esos niveles la emergencia de mecanismos cuasi-universales de procesamiento, de complejidad progresiva, que tienen como fin el "luchar" contra cualquier tipo de ausencia en el ciclo vital del agregado social—en la "sociedad informacional" - e igualmente en los ciclos individuales de los componentes.

Así pues, es un nuevo tipo de existencia, de duración en el tiempo, basado en la solución de las ausencias o "vacíos funcionales" (o simplemente problemas) de los ciclos vitales, tanto del agregado social como de los componentes individuales. Recordando el motto aristotélico de que "la naturaleza aborrece el vacío", la vida aparece desde esta perspectiva como una lucha perpetua contra la decadencia, contra las inevitables ausencias o vacíos funcionales. Lo cual no está en absoluto contrapuesto al anterior esquema de flujo vertical. El flujo aparece ahora como una compleja topología dinámica de "vacíos": vacíos funcionales (roturas de simetría) brotando de la dimensión molecular y percolando a través de células y organismos hacia la dimensión más alta de las sociedades; y lo mismo en la dirección opuesta (Matsuno, 1985, Marijuán, 1996). Se trata siempre de un inacabable proceso dinámico de equilibración y de desequilibrio, de generación de problemas y solución de los mismos. Y ello ocurre en todas las dimensiones posibles de descomposición, no sólo hacia arriba y hacia abajo, sino también horizontalmente; encontrando o no soluciones parciales por medio de los mecanismos cuasi-universales de procesamiento, y creando o no nuevas instancias de desequilibrio.

Este punto de vista no significa que la información sea simplemente "vacío", sino que es necesario clarificar en ella la diferencia entre señal o pauta y conteni$d o$; este último es el que corresponde a un genuino "vacío funcional" en una sociedad informacional (Marijuán, 1995, 1996). Al respecto, es curioso constatar

Scire. $2: 1$ (en.-jun. 1996). 
el amplio uso de los conceptos de "ausencia" y "vacío" en el mundo intelectual, y de la consiguiente actividad de "llenar" el vacío de que se trate (o sea poner en marcha los mecanismos cuasi-universales de detección y solución de problemas, como diríamos aquí). Leemos por ejemplo en una relevante obra del biólogo teórico Robert Rosen (1991): "Como ya he dicho, falta algo. Algo grande, pero es difícil ver incluso las cosas más grandes cuando precisamente están faltando. Tan sólo podemos apreciar el vacío de su ausencia e intentar fabricar lo que sea preciso para llenarlo... Esto proporcionará una manera de apreciar la forma del vacío que necesitamos llenar.” (pp. 14, 15)

Añadamos brevemente que no sólo el pensador o el científico puede apreciar "vacíos" y fabricar lo que es preciso para llenarlos; de hecho, desde la humilde bacteria hasta el más avanzado sistema nervioso se asiste a la emergencia de un continuo de mecanismos cuasi-universales dedicados a fabricar, generar los elementos funcionales precisos para llenar cualquier ausencia o vacío funcional a cualquier nivel organizativo. Los "motores" generativos de la célula (síntesis y degradación de proteínas) forman un continuo en relación con el "motor" del ciclo de reproducción y funcionamiento" del agregado multicelular, y de ahí podemos pasar fácilmente a la actividad neuro-muscular y al "trabajo" del organismo individual, o a la actividad en los sistemas socioeconómicos y políticos (De Vree, 1991). Todos estos sistemas están inmersos en organizaciones cuasiuniversales de solución de problemas. Atendamos también a que podemos relacionar las reorganizaciones estructurales derivadas de la solución de esos vacíos funcionales con la discusión del concepto de entropía o quizás mejor, con la entropía negativa de Schrödinger o la exponencial inversa de Stonier (véase Gil y Marijuán-este volumen). Pero ese fascinante tema de las relaciones entre información y entropía nos llevaría demasiado lejos, y no podemos ahora seguir con él ahora (Stonier, 1990; Swenson y Turvey, 1991; Matsuno, 1993; Gil y Marijuán—in press).

\section{Importancia de la conferencia: posibles repercusiones}

Muchos de los participantes en esta conferencia compartimos la intuición de que un positivo desenlace de la misma podría ser importante, en muy diversos sentidos. Dejando de lado los aspectos científicos particulares, me ceñiré brevemente a unos pocos aspectos más generales.

Contemplando al sistema de las ciencias como un todo unitario, quizás sea aquí, en el desarrollo de una perspectiva informacional, donde pueda encontrarse la clave conceptual para armonizar las construcciones de las ciencias naturales, las ciencias sociales y las humanidades. A través del puente de la información, por así decirlo, esas ramas separadas del árbol del conocimiento pueden reencontrarse nuevamente. La realización de esa convergencia no sólo represen-

Scire. 2 : 1 (en.-jun. 1996). 
ta una larga aspiración intelectual, sino también una necesidad hondamente sentida en el panorama actual. La armonía en el sistema de las ciencias aparece como un paso previo para afrontar con mayores garantías de éxito la solución de problemas a más alto nivel en la sociedad actual: problemas ecológicos, económicos, culturales, políticos. A medio plazo, no parece descabellado pensar que la supervivencia social podría depender del grado de coherencia del propio sistema de las ciencias.

El papel de las ciencias como instrumento social de conocimiento tiene un indudable paralelo con el procesamiento que realizan los sistemas nerviosos en relación con el conjunto del organismo. Las ciencias constituyen el aparato epistémico a más alto nivel de abstracción para la solución de los problemas existenciales de las sociedades humanas (la ciencia, particularmente la física, ha escrito Ortega es el "órgano de la felicidad humana"). Y de la misma manera que los sistemas nerviosos han tenido que inventar a lo largo del proceso evolutivo nuevas maneras de combinar e integrar la información especializada de sus distintas modalidades sensoriales de cara a la supervivencia del organismo, también las sociedades han precisado a lo largo de su evolución el desarrollo de adecuadas invenciones integrativas para recomponer un funcionamiento sistémico de las ciencias a partir de los contenidos especializados de las distintas disciplinas científicas. Es lamentable que las aburridas discusiones sobre reduccionismo, falsacionismo, relativismo, etc., hayan capturado la atención científico-filosófica durante estas décadas recientes, bloqueando el avance en lo que podemos denominar como "problema perspectivista" o "interdisciplinar". El hecho es que el análisis científico y filosófico contemporáneo es extremadamente pobre mostrando cómo las ciencias han de combinar e integrar sus herramientas especializadas.

En el siglo XX la ciencia, entendida en sentido amplio, ha pasado a ser un factor decisivo de los procesos de producción. Los científicos trabajan en instituciones especializadas, cada vez más parececidas en su estructura interna a grandes factorías y, junto con la burocracia educativa, constituyen una franja importante de las clases medias. El famoso ensayo de Ortega acerca de "la barbarie del especialismo" (1930) fue uno de los más tempranos avisos acerca de los riesgos de oscurantismo y degradación inherentes al proceso de estrecha especialización científica. También Whitehead (1925) había alertado acerca de la degeneración de la vida intelectual ante el imparable avance de la profesionalización y masificación del trabajo científico. Hoy día, no hace falta argumentar mucho al respecto, el oscurantismo de los científicos y tecnólogos fuera de su propia especialidad es bastante mayor que en aquellos tiempos. En líneas generales, la sociedad contemporánea ha continuado con el desarrollo de un sistema de conocimiento peligrosamente sesgado. Sorprende poco, pues, que la ciencia esté haciendo una escasa contribución a la solución de problemas al más alto nivel. Y que el pano-

Scire. $2: 1$ (en.-jun. 1996). 
rama de la acumulación social de problemas se vaya agravando con el paso del tiempo: superpoblación, crisis ecológica, violencia xenófoba, pobreza galopante, desorden internacional.

Pocos rechazarán que se hace necesario un profundo cambio en la orientación del conjunto de las instituciones educativas y científicas. Pero quizás previamente haya que desarrollar una nueva perspectiva que permita contemplar integralmente la interacción sociedad-conocimiento, es decir la solución social de problemas a la más amplia escala. Parece plausible que una ciencia de la información, bien desarrollada, contribuiría a iluminar nuestro uso global del conocimiento y permitiría avanzar en la solución nacional e internacional de problemas en esta que, paradójicamente, no deja de ser la "era de la información."

Siglos atrás fueron Erasmo, Vives, Ramus, More, Bacon..., quienes sintieron en su tiempo la necesidad de "nueva ciencia". Porque el antiguo modo de hacer ciencia era excesivamente estrecho y no estaba resolviendo los problemas de su tiempo. Lo mismo que entonces, necesitamos nueva ciencia ahora. Quizás la ciencia de la información pueda contribuir a abrir el sistema de las ciencias en las nuevas direcciones necesarias.

\section{Referencias bibliográficas}

Brookes, B. C. (1974). Robert Fairthorne and the scope of information science. // Journal of Documentation. $30: 2$ (1974) 139-152.

Conrad, M. (1984). Microscopic-Macroscopic Interface in Biological Information Processing. // BioSystems 16 (1984) 345-363.

Conrad, M. (1989). Physics and Biology : Towards a Unified Model. // Applied Mathematics and Computation 32 (1989) 75-102.

De Vree, J. K. (1991). Chaos in Europe: An inquiry into the nature of social systems and the methodology of the behavioral sciences. // Acta Politica $26: 1$ (1991) 25-63.

Horgan, J. (1995). From Complexity to Perplexity. // Scientific American. (June 1995) 7479.

Marijuán, P. C. (1995). Enzymes, artificial cells and the nature of biological information. BioSystems. 35 (1995) 167-170.

Marijuán, P. C. (1996). First Conference on Foundations of Information Science. // BioSystems. 38 (1996) 87-96.

Marijuán, P. C. (1996b). Gloom in the society of enzymes. // BioSystems. 38 (1996) 163171.

Matsuno, K. (1985). How can quantum mechanics of material evolution be possible? : Symmetry and symmetry-breaking in protobiological evolution. // Biosystems. 17 (1985) 179-192.

Matsuno, K. (1993). Being Free from Ceteribus Paribus : A Vehicle for Founding Physics on Biology Rather than the Other Way Around. // Applied Mathematics and Computation. 56 (1993) 261-279.

Scire. $2: 1$ (en.-jun. 1996). 


\section{8}

McLuhan, M. (1964). Understanding Media : The Extensions of Man. Toronto : University of Toronto Press, 1964. London : Ark Paperbacks, 1987.

Nagy, D. ; Horvath, P. ; Nagy, F. (1989). R. Ortway's letter to Von Neumann : The von Neumann-Ortvay Connection. // Annals of the History of Computing. 11 (1989) 183188.

Ortega y Gasset, J. (1930) La rebelión de las masas. Madrid : Diario el Sol, 1930.

Rosen, R. (1991) Life Itself: A Comprehensive Inquiry into the Nature, Origin, and Fabrication of Life. New York : Columbia University Press, 1991.

Scarrot, G. (1986) The need for a 'science' of information. // J. Inform. Technol. $1: 2$ (1986) 33-38.

Simon, H.A. (1969) The Sciences of the Artificial. Cambridge. MA : The MIT Press, 1969.

Stonier, T. (1990). Information and the Internal Structure of the Universe: An Exploration into Information Physics (Springer, London). Ed. española: Barcelona : Hacer, 1996.

Swenson, R. ; Turvey, M.T. (1991). Thermodynamic Reasons for Perception-Action Cycles. Ecological Psychology. 3 : 4 (1991) 317-348.

Wiener, N. (1948). Cybernetics : Or Control and Communication in the Animal and the Machine. Cambride, MA : The MIT Press, 1948.

Wigner, E. V. (1989). Personal interview. // Brink, J. R. ; Haden, C. R. ; Burawa, C. (eds.).The Computer and the Brain: Perspectives on Human and Artificial Intelligence. New York : North Holland, 1989.

Whitehead, A.N. (1925). Science and the Modern World. New York : The Free Press, Macmillan Pub.Co., N Y, 1925.

Whitehead, A.N. (1948). An Introduction to Mathematics. New York : Oxford University Press, 1958. 